NASA Technical Memorandum 102330

ICOMP-89-20

\title{
Numerical Investigation of an Internal Layer in Turbulent Flow Over a Curved Hill
}

S.-W. Kim

Institute for Computational Mechanics in Propulsion

Lewis Research Center

Cleveland, Ohio

October 1989

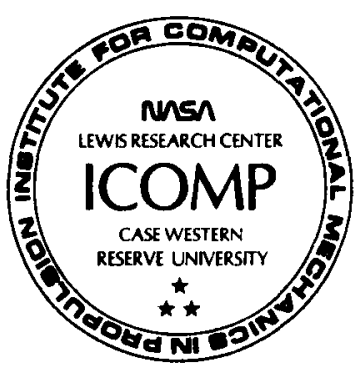

(NASA-TM-102330) NIJMERTCAL INVESTIGATION UF AN INTERNAL LAYER IN TURBULFNT FLOW OVER A CURVEO HILL (NASA. LeWIS Research Center) $44 \mathrm{D}$ CSCL 2 On

N89-29725 
NUMER ICAL INVESTIGATION OF AN INTERNAL LAYER IN

TURBULENT FLOW OVER A CURVED HILL

\author{
S. -W. Kim* \\ Institute for Computational Mechanics in Propulsion \\ Lewis Research Center \\ Cleveland, Ohio 44135
}

\title{
SUMMARY
}

The development of an internal layer in a turbulent boundary layer flow over a curved hill is investigated numerically. The turbulence field of the boundary layer flow over the curved hill is compared with that of a turbulent flow over a symmetric airfoil (which has the same geometry as the curved hill except that the leading and trailing edge plates were removed) to study the influence of the strongly curved surface on the turbulence field. The turbulent flow equations are solved by a control-volume based finite difference method. The turbulence is described by a multiple-time-scale turbulence model supplemented with a near-wall turbulence model. Computational results for the mean flow field (pressure distributions on the walls, wall shearing stresses and mean velocity profiles), the turbulence structure (Reynolds stress and turbulent kinetic energy profiles), and the integral parameters (displacement and momentum thicknesses) compared favorably with the measured data. Computational results show that the internal layer is a strong turbulence field which is developed beneath the external boundary layer and is located very close to the wa11. Development of the internal layer was more obviously observed in the Reynolds stress profiles and in the turbulent kinetic energy profiles than in the mean velocity profiles. In this regard, the internal layers is

*Work funded by Space Act Agreement C99066G. 
significantly different from wall-bounded simple shear layers in which the mean velocity profile characterizes the boundary layer most distinguishably. Development of such an internal layer, characterized by an intense turbulence field, is attributed to the enormous mean flow strain rate caused by the streamline curvature and the strong pressure gradient. In the turbulent flow over the curved hill, the internal layer begin to form near the forward corner of the hill, merges with the external boundary layer, and develops into a new fully turbulent boundary layer as the fluid flows in the downstream direction. For the flow over the symmetric airfoil, the boundary layer began to form from almost the same location as that of the curved hill, grew in its strength, and formed a fully turbulent boundary layer from mid-part of the airfoil and in the downstream region. Computational results also show that the detailed turbulence structure in the region very close to the wall of the curved hill is almost the same as that of the airfoil in most of the curved regions except near the leading edge. Thus the internal layer of the curved hill and the boundary layer of the airfoil were also almost the same. Development of the wall shearing stress and separation of the boundary layer at the rear end of the curved hill mostly depends on the internal layer and is only slightly influenced by the external boundary layer flow. 


\section{INTRODUCTION}

Turbulent flows subjected to various strain rates (in addition to the simple shearing strain rates) caused by streamline curvature, strong pressure gradient, separation and reattachment, swirl velocity and interaction of multiple number of shear layers are usually called "complex turbulent flows." The turbulence structure of such complex turbulent flows is more complicated than that of simple shear layer flows. Calculations of complex turbulent flows using various turbulence models such as the k- $\epsilon$ turbulence models, algebraic Reynolds stress turbulence models (ARSM), and Reynolds stress turbulence models (RSM) yield rather unsatisfactory computational results (Kline, Cantwell\& Lilley 1982). Many turbulence models, improved by modifying the standard form turbulence equations (usually, the dissipation rate equation for $k-\epsilon$ and ARSM and the pressure-strain correlation term for RSM) to yield better computational results for a few flow cases, have produced worse agreement with the measured data than the standard turbulence models for other classes of turbulent flows (Persen 1986). In recent years, a number of efforts have been made experimentally to better understand complex turbulent flows by separating the mechanisms that produce the extra strain rates. For example, experimental investigations of turbulent flows in curved channels (Gillis \& Johnston 1983; So \& Mellor 1973) and turbulent flows over a curved hill (Baskaran, Smits \& Joubert 1987) were made to study the effect of streamline curvature on turbulence structure.

Turbulent shear layers over curved surfaces are highly sensitive even to a small amount of streamline curvature (Bradshaw 1969 \& 1973). Bradshaw (1969) proposed a curvature correction method based on an assumption that such turbulent flows can be characterized by a "curvature parameter", that 
is, the ratio of boundary layer thickness $(\delta)$ to radius of curvature (R). In the curvature correction method, the mixing length is altered by a factor (F) given as;

$$
F=1-\beta \frac{U / r}{\partial U / \partial n}
$$

where $U$ is the streamwise velocity, $n$ is the coordinate normal to the streamline, $r$ is the radius of curvature of the local streamline, and $\beta$ is a constant coefficient. Application of the method to a number of turbulent flows with varying streamline curvatures revealed that the constant coefficient $(\beta)$ needs to be adjusted for different flows to obtain computational results in good agreement with the measured data. To take into account the varying coefficient $(\beta)$ for turbulent flows with different streamline curvature, a functional form of $\beta$ which depends on the curvature Richardson number was also proposed (Gibson 1978). Many turbulence models incorporating a curvature correction method yield improved computational results for turbulent flows over mildly curved surfaces and the computational results help to better understand the turbulence structure of such flows; however, these turbulence models still fail to predict the turbulence field for turbulent flows with large streamline curvature. A discussion on the shortcomings of various curvature correction methods can be found in Gibson, Jones \& Younis (1981). Gillis \& Johnston (1983) found that turbulent flow over a curved surface reaches a "saturated state" as the streamline curvature is further increased and that many turbulence models did not yield meaningful results for such flows. Baskaran, Smits \& Joubert (1987) presented experimental data for a turbulent boundary layer over a curved hill and a turbulent flow over a symmetric airfoil. The 
curved hill and the airfoil shared the same geometry. They found that an internal boundary layer is formed beneath the external boundary layer over the curved hill and that the internal layer shares many similarities with the boundary layer flow over the symmetric airfoil. The experimental data also showed that the internal layer was insensitive to the external boundary layer and the curvature parameter $(\delta / R)$. It was also suggested in Baskaran, Smits \& Joubert (1987) that the effects of prolonged convex curvature can be more accurately represented by the ratio of the internal layer thickness to the surface radius of curvature than by the conventional curvature parameter. Here, detailed computational results for the turbulent boundary layer flow over the curved hill and the turbulent flow over the symmetric airfoil obtained using a multiple-time-scale turbulence model (Kim \& Chen 1987; Kim 1988) are presented. The multiple-time-scale turbulence model (hereafter abbreviated as the M-S turbulence model), the near-wall turbulence model and the numerical method used herein are introduced below.

It has been shown previously that the high Reynolds number M-S turbulence model yields accurate computational results for a number of complex turbulent flows. The wall-jet flow (Irwin 1973) and the wake-boundary layer interaction flow (Tsiolakis, Krause \& Muller 1983) involve interaction of two turbulent shear layers. It is shown in Kim \& Chen (1987) that the M-S turbulence model yields accurate computational results for these flows. It is also shown in Kim \& Chen (1987) that the computational results for a backward-facing step flow (Kim, Kline \& Johnston 1980) obtained using the M-S turbulence model compared somewhat more favorably with the measured data than those obtained using a Reynolds stress turbulence model. In the confined coaxial swirling jet (Roback \& 
Johnson 1983), the axial velocity in the center region is retarded by the influence of the swirl velocity and a large reversed flow region is formed in the core region of the flow. Due to the existence of the reversed flow region, which is often referred to as a "flame stabilizer" in combustors, the flow is subjected to a large amount streamline curvature. This flow also contains a separated and reattaching shear layer in the corner region. It can be found in Kim \& Chen (1987) that the M-S turbulence model yields significantly improved computational results compared with those obtained using the standard $k-\epsilon$ turbulence models. The experimental study of a reattaching shear layer in a divergent channel (Driver \& Seegmiller 1985) was primarily designed to study the effect of pressure gradient on the development of Reynolds stresses and the reattaching shear layer, to identify any deficiency in turbulence closure models, and thus to improve predictive capability of turbulence models. A number of turbulence models, such as the $k-\epsilon$ turbulence models and algebraic Reynolds stress turbulence models (ARSM), were shown to yield poor computational results for the flow (Driver \& Seegmiller 1985). It was also shown that a modified ARSM yielded computational results which are in good agreement with measured data. However, generality of the improved predictive capability of the turbulence model for other complex turbulent flows has not been shown yet. On the other hand, the computational results obtained using the M-S turbulence model compared somewhat more favorably with the measured data than those obtained using the modified ARSM. Recall that the turbulent transport of mass and momentum is governed by the time scale of the energy containing large eddies and the dissipation of the turbulent kinetic energy is governed by the time scale of the fine scale eddies (Lumley 1983). In M-S turbulence models (Hanjelic, Launder \& Schiestel 1980; Kim \& Chen 1987; Kim 
1988), the turbulent transport of mass and momentum is described using the time scale of the large eddies and the dissipation rate is described using the time scale of the fine-scale eddies. In this regard, a well posed M-S turbulence model can describe the turbulent transport process better than the single-time-scale turbulence models such as the $k-\epsilon$, ARSM, and RSM turbulence models. Single-time-scale turbulence models yield reasonably accurate computational results for simple turbulent flows; however, the predictive capability degenerates rapidly as turbulent flows to be solved become more complex. This nature may due to the use of a single time scale to describe both the turbulence transport mechanism and the dissipation rate.

In numerical calculations of turbulent flows, wall function methods are most frequently used to model the near-wall region. The wall function methods have been derived from a logarithmic velocity profile which usually prevails in wall-bounded simple shear layer flows. The wall function methods are not valid if the logarithmic velocity profile no longer prevails in the near-wall region. For the turbulent flows considered herein, a strong inequilibrium turbulence field is formed in the region very close to the wall. Such a turbulence field can not be described adequately by the wall function methods. Many other cases for which the wall function methods become invalid can be found in Kim (1988). A detailed discussion on various approaches to be used in place of wall function methods, including their advantages and disadvantages, can also be found in Kim (1988). In the near wall turbulence model used herein, the turbulent kinetic energy equations are extended to include the near-wall low turbulence region and the energy transfer rate and the dissipation rate inside the near-wall layer are obtained from algebraic equations. The 
algebraic equations were obtained from a k-equation turbulence model (Wolfshtein 1969). It would be appropriate to classify the method as a "partially low Reynolds number approach" to distinguish it from other classes of methods. This approach was first used in Chen and Patel (1987) to solve turbulent flows over airfoils. Recall that the turbulence length scale in the region very close to a wall is related to the normal distance from the wall while that of external flows is related to the flow field characteristics (Roshko 1976). This characteristic of wall bounded turbulent flows can be described quite accurately by the "partially low Reynolds number turbulence models." Development of the near-wall turbulence model and its application to fully developed turbulent channel and pipe flows can be found in Kim (1988). It has been shown in the reference that the present near-wall turbulence model can resolve the over-shoot phenomena of the turbulent kinetic energy and the dissipation rate in the region very close to the wall and that significantly improved computational results for the turbulence structure in the near-wall region are obtained. It is also interesting to note that a similar k-equation turbulence model, which forms the basis of the present near-wall turbulence model, yields fairly accurate computational results for a fully developed unsteady turbulent pipe flow (Tu \& Ramaprian 1983). Incorporation of the same near-wall turbulence model into a k- $\epsilon$ turbulence model and its application to a yet different class of complex turbulent flows such as a supersonic turbulent flow over a compression ramp and a transonic flow over an axisymmetric curved hill can be found in Kim (1989a; 1989b).

The numerical method used herein is based on the pressure correction method (Patankar 1980) which has been used most extensively to solve incompressible flows. However, the present numerical method is applicable 
for both incompressible and compressible flows with arbitrary, complex geometries. The capability to solve compressible flows is achieved by including a convective incremental pressure term into the pressure correction equation (Kim 1989a). The accuracy and the convergence nature of the numerical method have been demonstrated by solving a number of flow cases. The example problems considered in Kim (1989a; 1989b) include: developing channel and pipe flows, a two-dimensional laminar flow in a 90 degree bent channel, polar cavity flows, a turbulent supersonic flow over a compression ramp, and a shock wave - turbulent boundary layer interaction in transonic flow over a curved hill.

\section{TURBULENCE EQUATIONS}

As introduced in the previous section, the M-S turbulence model yields accurate computational results for a number of complex turbulent flows subjected to various different types of extra strains. However, the model is relatively new and is discussed in some detail below.

The M-S turbulence model is based on a simplified split-spectrum method (Hanjelic, Launder \& Schiestel 1980). The transport equations for the turbulent kinetic energy of large eddies $\left(k_{p}\right)$ and that of fine scale eddies $\left(k_{t}\right)$ are given as;

$$
\begin{aligned}
& u_{j} \frac{\partial k_{p}}{\partial x_{j}}-\frac{\partial}{\partial x_{j}}\left(\left(\nu+\frac{\nu_{t}}{\sigma_{k p}}\right) \frac{\partial k_{p}}{\partial x_{j}}\right)=\operatorname{Pr}-\epsilon_{p} \\
& u_{j} \frac{\partial k_{t}}{\partial x_{j}}-\frac{\partial}{\partial x_{j}}\left(\left(\nu+\frac{\nu_{t}}{\sigma_{k t}}\right) \frac{\partial k_{t}}{\partial x_{j}}\right)=\epsilon_{p}-\epsilon_{t}
\end{aligned}
$$


where $\operatorname{Pr}$ is the production rate of turbulent kinetic energy, and $\epsilon_{p}$ and $\epsilon_{t}$ are the energy transfer and dissipation rates, respectively. Eqs. (1-2) imply that the turbulent kinetic energy is generated by the instability of the mean fluid motion, is transferred to the high wave number region, and is dissipated by the molecular viscosity of the fluid. This mathematical model is consistent with the physically observed evolution of the turbulent kinetic energy (Tennekes \& Lumley 1972) except that the cascade process of the turbulent kinetic energy is over-simplified and is represented by the single energy transfer rate. This over-simplification is still better justified than the single-time-scale turbulence models if one considers that only the generation and dissipation of turbulent kinetic energy are considered in the latter classes of turbulence models.

The energy transfer rate and the dissipation rate away from the near-wall region are given as;

$$
\begin{aligned}
& \left.\mathrm{u}_{j} \frac{\partial \epsilon_{\mathrm{p}}}{\partial \mathrm{x}_{\mathrm{j}}}-\frac{\partial}{\partial \mathrm{x}_{\mathrm{j}}}\left(\nu+\frac{\nu_{\mathrm{t}}}{\sigma_{\epsilon \mathrm{p}}}\right) \frac{\partial \epsilon_{\mathrm{p}}}{\partial \mathrm{x}_{\mathrm{j}}}\right)=\mathrm{c}_{\mathrm{p} 1} \frac{\operatorname{Pr}^{2}}{\mathrm{k}_{\mathrm{p}}}+\mathrm{c}_{\mathrm{p} 2} \frac{\operatorname{Pr} \epsilon_{\mathrm{p}}}{\mathrm{k}_{\mathrm{p}}}-\mathrm{c}_{\mathrm{p} 3} \frac{\epsilon_{\mathrm{p}} \mathrm{k}_{\mathrm{p}}}{\mathrm{k}^{2}} \\
& u_{j} \frac{\partial \epsilon_{t}}{\partial x_{j}}-\frac{\partial}{\partial x_{j}}\left\{\left(\nu+\frac{\nu_{t}}{\sigma_{\epsilon t}}\right) \frac{\partial \epsilon_{t}}{\partial y}\right\}=c_{t 1} \frac{\epsilon_{p}^{2}}{k_{t}}+c_{t 2} \frac{\epsilon_{p} \epsilon_{t}}{k_{t}}-c_{t 3} \frac{\epsilon_{t}{ }^{2}}{k_{t}}
\end{aligned}
$$

where the load functions in eqs. (3-4) were obtained from a physical dimensional analysis, and $c_{p \ell}$ and $c_{t \ell}(\ell=1,3)$ are the model constants. These model constants were obtained by solving a five by five system of equations and by numerically optimizing one model constant $\left(c_{t 1}\right)$ to yield the best solution for a fully developed channel flow (Laufer 1949) and a plane jet exhausting into a moving stream (Bradbury 1965). One equation for 
the model constants has been obtained from the equilibrium turbulent flow condition. Two equations have been obtained by transforming the multiple-time-scale turbulence equations into asymptotic turbulence growth rate equations which are equivalent to that of Harris, Graham \& Corrsin (1977). The other two equations have been obtained by transforming the present turbulence equations into asymptotic turbulence decay rate equations which are equivalent to that given in Harlow and Nakayama (1968). The turbulence model constants are given as; $\sigma_{\mathrm{kp}}=0.75, \sigma_{\epsilon \mathrm{p}}=1.15, \sigma_{\mathrm{kt}}=0.75$, $\sigma_{\epsilon t}=1.15 . c_{p 1}=0.21, c_{p 2}=1.24, c_{p 3}=1.84, c_{t 1}=0.29, c_{t 2}=1.28$, and $c_{t 3}=1.66$. Further discussion on the establishment of these turbulence model constants can be found in Kim \& Chen (1987).

The eddy viscosity away from the near-wall region is given as;

$$
\nu_{t}=c_{\mu f} k^{2} / \epsilon_{p}=c_{\mu} k^{2} / \epsilon_{t}
$$

where $c_{\mu f}(=0.09)$ is a constant coefficient and $c_{\mu}=c_{\mu f} \epsilon_{t} / \epsilon_{p}$ is the effective eddy viscosity coefficient. In this equation, the turbulence length scale is related to the energy transfer rate of the energy containing large eddies rather than the dissipation rate of the fine-scale eddies. Experimental data show that the effective eddy viscosity coefficient $\left(c_{\mu}\right)$ decreases as the ratio $\mathrm{Pr} / \epsilon_{t}$ increases and increases as the same ratio decreases. The present $M-S$ turbulence model yields the solution in such a way that: $\operatorname{Pr}>\epsilon_{\mathrm{p}}>\epsilon_{\mathrm{t}}$ and $c_{\mu}<c_{\mu f}$ for $\mathrm{P}_{\mathrm{r}}>\epsilon_{\mathrm{t}} ; \mathrm{P}_{\mathrm{r}}=\epsilon_{\mathrm{p}}=\epsilon_{\mathrm{t}}$ and $c_{\mu}=c_{\mu f}$ for $P_{r}=\epsilon_{t} ;$ and $\epsilon_{p}<\epsilon_{t}$ and $c_{\mu}>c_{\mu f}$ for $P_{r} \ll \epsilon_{t}$. Thus the eddy viscosity given as eq. (5) yields a variation of the $c_{\mu}$ which is in good agreement with that observed in experimental data as well as that of the generalized algebraic stress turbulence models (Rodi 1972; Launder 1982; Kim \& Chen 
1988).

The eddy viscosity equation proposed by Hanjelic et al., which is given as $\nu_{t}=c_{\mu}\left(k_{p}+k_{t}\right) k_{p} / \epsilon_{p}$, is compatible with near wall mixing length theory or with the standard near-wall analysis only when $k_{t}$ vanishes in the near wall region. On the other hand, the eddy viscosity given in eq. (5) is compatible with both the near wall mixing length theory and the wall function method.

The energy transfer rate and the dissipation rate inside the near-wall layer are given as;

$$
\epsilon_{\mathrm{p}}=\epsilon_{\mathrm{t}}=\frac{\epsilon_{1}}{f_{\epsilon}}
$$

where $\epsilon_{1}=c_{\mu f} f^{3 / 4} k^{3 / 2} / \kappa y$ is the standard dissipation rate for near-wall turbulent flows in equilibrium state, $f_{\epsilon}-1-\exp \left(-A_{\epsilon} R_{t}\right)$ is a wall damping function, $R_{t}-k^{2} / \nu \epsilon_{1}$ is a turbulent Reynolds number, and $A_{\epsilon}=c_{\mu f} f^{3 / 2} / 2 \kappa^{2}$ is a constant coefficient. The wall damping function was constructed in such a way that eq. (6) takes a limit value of $2 \nu \mathrm{k} / \mathrm{y}^{2}$ for $\mathrm{y} \approx 0$ and $\mathrm{f}_{\epsilon}$ becomes unity in the region slightly away from the wall where the turbulence is in equilibrium state. For near-wall equilibrium turbulent flows, the production rate is approximately equal to the dissipation rate $\left(\epsilon_{t}\right)$ and hence the energy transfer rate $\left(\epsilon_{\mathrm{p}}\right)$ from the low wave number production range to the high wave number dissipation range has to be approximately equal to both of them. Recall that the production rate vanishes on the wall and grows to a peak value at the wall coordinate $\left(\mathrm{y}^{+}\right)$approximately equal to 15. Hence eq. (6) may not be a good approximation for $0<y^{+}<15$. However, use of the vanishing boundary condition for the turbulent kinetic energy on the wall yields a growth rate of turbulent kinetic energy and a production 
rate that are in good agreement with experimental data as well as with theoretical analysis (Kim 1988).

The eddy viscosity in the near-wall layer is given as;

$$
\nu_{t}=c_{\mu f} f_{\mu} \frac{k^{2}}{\epsilon_{1}}
$$

where $f_{\mu}=1-1 . / \exp \left(A_{1} \sqrt{ } R_{t}+A_{2} R_{t}^{2}\right)$ is a linear function of the distance from the wall in the viscous sublayer and becomes unity in the fully turbulent region. $A_{1}=0.025$ and $A_{2}=0.00001$ have been used for the near wall layer (see Kim 1988). The eddy viscosity given as eq. (7) grows in proportion to the cubic power of the distance from the wall. It can be found in Kim (1988) that the near-wall analysis yields the same growth rate of the eddy viscosity in the region very close to the wall. The improved predictive capability of the present turbulence model is attributed to the use of the multiple-time-scales, the physically consistent eddy viscosity equation, and the near-wall turbulence model.

\section{NUMERICAL METHOD}

The incompressible turbulent flow equations are given as;

$$
\frac{\partial}{\partial \mathrm{x}}(\rho \mathrm{u})+\frac{\partial}{\partial \mathrm{y}}(\rho \mathrm{v})=0
$$

$$
\frac{\partial}{\partial \mathrm{x}}(\rho \mathrm{uu})+\frac{\partial}{\partial \mathrm{y}}(\rho \mathrm{uv})=\frac{\partial}{\partial \mathrm{x}}\left(2 \mu_{\mathrm{e}} \underset{\partial \mathrm{x}}{-}\right)+\frac{\partial}{\partial \mathrm{y}}\left(\mu_{\mathrm{e}}\left(\frac{\partial \mathrm{u}}{\partial \mathrm{y}}+\frac{\partial \mathrm{v}}{\partial \mathrm{x}}\right)\right)-\frac{\partial \mathrm{p}}{\partial \mathrm{x}}
$$




$$
\frac{\partial}{\partial x}(\rho \mathrm{uv})+\frac{\partial}{\partial \mathrm{y}}(\rho \mathrm{vv})=\frac{\partial}{\partial \mathrm{x}}\left\{\mu_{\mathrm{e}}\left(\frac{\partial \mathrm{u}}{\partial \mathrm{y}}+\frac{\partial \mathrm{v}}{\partial \mathrm{x}}\right)\right\}+\frac{\partial}{\partial \mathrm{y}}\left(2 \mu_{\mathrm{e}} \frac{\partial \mathrm{v}}{\partial \mathrm{y}}\right)-\frac{\partial \mathrm{p}}{\partial \mathrm{y}}
$$

where $\mathrm{x}$ and $\mathrm{y}$ are spatial coordinates, $\mathrm{u}$ and $\mathrm{v}$ are cartesian velocities, $\rho$ is the density, $\mu_{\mathrm{e}}\left(-\mu+\mu_{t}\right)$ is the effective viscosity, $\mu$ is the molecular viscosity, $\mu_{t}$ is the turbulent viscosity, and eqs. $(8-10)$ follow from the conservation of mass, $u$-momentum, and v-momentum, respectively. In numerical calculation of incompressible flows, the conservation of mass equation is replaced by a pressure correction equation given as:

$$
\frac{\partial}{\partial \mathrm{x}}\left(\rho^{*} \mathrm{~A}_{\mathrm{u}} \frac{\partial \mathrm{p}^{\prime}}{\partial \mathrm{x}}\right)+\frac{\partial}{\partial \mathrm{y}}\left(\rho^{*} \mathrm{~A}_{\mathrm{v}} \frac{\partial \mathrm{p}^{\prime}}{\partial \mathrm{y}}\right)=\nabla \cdot\left(\rho^{*} \mathrm{v}^{*}\right)
$$

where the superscript $(*)$ denotes the current values, $A_{u}$ and $A_{v}$ are coefficients relating the incremental velocities and the incremental pressure and the last term represents the mass imbalance. A similar pressure correction equation for both incompressible and compressible flows and its derivation can be found in Kim (1989a). In the present numerical method, the velocities are located at the same grid points and the pressure is located at the centroid of the cell formed by the four adjacent velocity grid points. Any other flow variables such as the turbulent kinetic energy and the dissipation rate are located at the velocity grid points.

The control volume for the pressure correction equation is defined as the cell enclosed by the four neighboring grid points. The velocity-pressure decoupling is eliminated by treating the pressure correction equation as a continuous form partial differential equation (hereafter, abbreviated as p.d.e.) rather than treating it as a constraint 
condition at each grid point. In the former case, the discrete pressure correction obtained from eq. (11) becomes a five-diagonal system of equations for rectangular grids. The off-diagonal terms are contributed only by the non-orthogonal grids and their magnitude is much smaller than that of the diagonal terms even for highly skewed grids. On the other hand, in the latter method (Vanka, Chen \& Sha 1980), the discrete pressure correction equation, obtained by directly substituting the incremental pressure - incremental velocity relations into the conservation of mass equation, yields a nine-diagonal system of equations for orthogonal grids. This discrete pressure correction equation can yield a velocity-pressure decoupled solution, whereas the former equation does not. The present pressure correction equation is more like that of Braza, Chassaing \& Ha Minh (1986) than the more standard pressure correction equation (Vanka, Chen \& Sha 1980) in the sense that the equation is dealt as a continuous form p.d.e. In solving the discrete system of equations, the off-diagonal terms are moved to the load vector term and the resulting system of equations can be solved using a tri-diagonal matrix algorithm (TDMA). Incorporating the off-diagonals into the load vector term did not degenerate the convergence rate (Kim 1989a).

In control-volume methods, the discrete system of equations is derived by integrating the governing differential equations over the control volume. For curvilinear grids, the required number of interpolations to obtain flow variables at the cell boundaries is significantly reduced by using the present grid layout. The enhanced convergence rate, even when highly skewed, unequally spaced, curved grids are used, is attributed to the pressure correction equation cast in a continuous form p.d.e. and the grid layout which required fewer interpolations. As shown in this paper, 
the method yields accurate computational results (i.e., free of numerical wiggles) for grid aspect ratios as large as a few thousand. The pressure correction equation cast in a continuous form p.d.e. is undoubtedly more advantageous than the more standard pressure correction equation (Vanka, Chen \& Sha 1980) for the reasons discussed above.

\section{COMPUTATIONAL RESULTS}

The turbulent flow over a curved hill is schematically shown in Figure 1. The chord length (C) and the height $(H)$ of the curved hill and the airfoil are 1.284 meters and 0.208 meters, respectively. The unit Reynolds number based on the free stream velocity $\left(U_{\infty}-20 \mathrm{~m} / \mathrm{sec}\right)$ is $1.33 \times 10^{6} / \mathrm{meter}$. In external flows, the location of the far field boundary and the boundary conditions specified at the far field can influence the computational results. In the present study, the dependence of the computational results on the grid size, the location of the far field boundary, and the boundary conditions prescribed at the far field boundaries has also been studied.

In the experimental investigation of the turbulent flow over the curved hill, the flow was made turbulent using a trip wire located $0.65 \mathrm{C}$ upstream of the forward corner of the curved hill. Hence in the numerical calculations, the upstream boundary $\left(\partial \Omega_{1}\right)$ was located $0.61 \mathrm{C}$ upstream of the forward corner of the curved hill. Inclusion of the trip wire in numerical calculation of the entire flow field is prohibitive at present due to the limitation imposed by the computational resources. Thus, the inlet boundary conditions for the tangential velocity, the turbulent kinetic energies, and the dissipation rates $\left(\epsilon_{p}\right.$ and $\epsilon_{t}$ ) were obtained from experimental data for a fully developed boundary layer flow over a flat plate (Klebanoff 1955). The non-dimensional velocity and the turbulent kinetic energy profiles were 
scaled to yield a boundary layer thickness of 0.044 meters at the inlet boundary. This inlet boundary condition is somewhat different from that of the tripped turbulent flow; however, it is considered to be a reasonable approximation to the tripped turbulent flow since development of the internal layer is less dependent on the external boundary layer flow as can be found in the following discussion. In a series of numerical tests for the flow over the curved hill, the downstream boundary was located at $1.4 \mathrm{C}$ and $1.9 \mathrm{C}$ downstream of the rearward corner of the hill, and the top boundary was located at $9.3 \mathrm{H}, 12.3 \mathrm{H}$, and $15.9 \mathrm{H}$ away from the wall, respectively. The computational results were not sensitive to the location of the downstream boundary as long as it is located reasonably far away from the reversed flow region. Location of the top boundary $\left(\partial \Omega_{2}\right)$ influenced the computational results substantially. For the latter two cases (e.g., $12.3 \mathrm{H}$ and $15.9 \mathrm{H}$ ), the computational results in the near-wall region were virtually the same and those in the far region differed by no more than one percent. The computational results presented herein were obtained with $\partial \Omega_{2}$ and $\partial \Omega_{3}$ located at $15.9 \mathrm{H}$ and $1.4 \mathrm{C}$ away from the wall and the rearward corner, respectively. The boundary conditions tested are:

(1) Dirichlet boundary condition (DBC) for $u$ and $v$ along $\partial \Omega_{2}$; and vanishing gradient boundary condition ( $\mathrm{NBC}$ ) for $u$ and $v$ and uniform pressure along $\partial \Omega_{3}$

and

(2) NBC for $u$ and $v$ and uniform pressure along $\partial \Omega_{2}$ and $\partial \Omega_{3}$.

In each case, $u=v=k_{p}=k_{t}=0$ was specified on the solid wall boundary $\left(\partial \Omega_{4}\right)$ and the NBC for $k_{p}, k_{t}, \epsilon_{p}$, and $\epsilon_{t}$ was specified along $\partial \Omega_{2}$ and $\partial \Omega_{3}$. The computational results obtained using the two different sets of boundary 
conditions were virtually the same when the external boundary $\left(\partial \Omega_{2}\right.$ and $\left.\partial \Omega_{3}\right)$ was located far enough so that the extent of the external boundary does not influence the computational result. A part of these computational results are also shown in the following.

For the turbulent flow over the symmetric airfoil, the far field boundaries were initially separated by the same distance from the airfoil as in the curved hill flow. Then the distances of the upstream $\left(\partial \Omega_{1}\right)$ and the downstream $\left(\partial \Omega_{3}\right)$ boundaries were increased by approximately $0.5 \mathrm{C}$, respectively, until the computational results became independent of the extent of the external boundary. The computational results presented herein were obtained with the upstream $\left(\partial \Omega_{1}\right)$ and the downstream $\left(\partial \Omega_{3}\right)$ boundaries separated by $2.1 \mathrm{C}$ and $3.1 \mathrm{C}$ from the forward and the rearward corners of the airfoil, respectively. Uniform flow velocities were prescribed at the upstream boundary. In the experiment, the flow was made turbulent using a trip wire installed at the leading edge region of the airfoil. However, inclusion of the trip wire in the numerical calculation of the entire flow is not feasible for the reason discussed previously. Hence in the numerical calculation, a small amount of turbulent kinetic energy $\left(k-2.5 \times 10^{-4} U_{\infty}{ }^{2}\right)$ and a dissipation rate $\left(\epsilon_{\mathrm{t}}=40 \mathrm{~m}^{2} / \mathrm{sec}^{3}\right)$ were prescribed at the inlet boundary so that the flow became fully turbulent from the leading edge region of the airfoil. For this boundary condition, the turbulent viscosity at the inlet boundary is approximately equal to the kinematic viscosity of the fluid. The boundary conditions along the $\partial \Omega_{2}$ and $\partial \Omega_{3}$ boundaries were the same as the first case of the curved hill flow. A symmetric boundary condition was used for all flow variables along the symmetry line in front of and behind the airfoil. Boundary conditions along the airfoil were the same as those used in the flow over the curved hill. 
A number of meshes were also tested in conjunction with the domain independence study to obtain grid independent solutions. In each case, the discrete finite difference system of equations was solved iteratively until the relative error for all flow variables became less than the prescribed convergence criterion $\left(5 \times 10^{-5}\right)$. A $143 \times 77$ mesh for the flow over the curved hill and a $146 \times 77$ mesh for the flow over the airfoil are shown in Figure 2 . The grid spacings transverse to the flow direction are the same in each case. The average grid size $\left(\Delta y^{+}\right)$to the first grid point from the wall is approximately 0.8 (i.e., $\Delta n=2 \times 10^{-5}$ meters). The grid size in the normal direction was increased by a factor of 1.15 approximately. The partition between the near-wall layer and the external region was located at $y^{+} \approx 200$ and 27 grid points were used inside the near-wall layer. The M-S turbulence model predicted the reversed flow regions at the rear end of the curved hill and the airfoil for all the cases tested in the present study. All the computational results presented herein, except the streamline contours shown in Figure 3, were obtained using the fine mesh.

The streamline contours for the flows over the curved hill and the airfoil obtained using the medium mesh ( $92 \times 60$ grid points in each case) are shown in Figure 3 . For the medium mesh, the average grid size $\left(\Delta y^{+}\right)$to the first grid point from the wall was 80 (i.e., $\Delta n=0.001$ meters). The partition between the near-wall layer and the external domain was located at $\mathrm{y}^{+} \approx 300$ and $3 \mathrm{grid}$ points were allocated inside the near wall layer. The calculated mean velocity, pressure, and turbulence fields were in fair agreement with the measured data.

The calculated static pressure distributions on the walls of the curved hill and the symmetric airfoil are compared with the measured data in Figure 4, where the pressure coefficients $\left(c_{p}\right)$ were obtained by 
normalizing the pressure distributions by the free stream dynamic pressure $\left(0.5 \rho \mathrm{U}_{\infty}^{2}\right)$. The calculated static pressure distribution along the symmetry line of the entire computational domain is also shown in the upper right corner of the figure. It can be seen from this figure that the numerical method does not yield an unphysical oscillatory solution for the mesh with the grid aspect ratio as large as a few thousand. Note that the measured data exhibit pressure jumps at the plateau of the hill and the airfoil. The pressure gradients caused by these jumps are believed to be strong enough to disturb the mean flow field and the disturbed mean velocity may leave some evidence in the measured wall shearing stress, at least slightly; however, such an evidence is not found in the measured wall shearing stress. It is not clear if these pressure jumps really existed or were caused by the interference of the wind tunnel wall or the measuring device. The cause of such pressure jumps was not clarified in Baskaran, Smits \& Joubert (1987). Such pressure jumps were not detected in the present calculations. It can be seen in the figure that the calculated pressure distributions compare favorably with the measured data, in general. The pressure distributions on the curved hill obtained using the two different boundary conditions are almost the same and almost collapse into a single line as shown in this figure. This indicates that the far field boundary condition can be implemented in one way or another as long as it is located far away from the wall. Also note that the measured pressure distribution on the wall of the airfoil is somewhat higher than the calculated result. This discrepancy might have been caused by the interference of the wall and/or measuring device in the experiments. This fact is further clarified in the following by comparing the measured and the calculated mean velocity profiles. 
The calculated wall shearing stresses for the curved hill and the airfoil are shown in Figures 5 and 6 , respectively, where the friction coefficients $\left(c_{f}\right)$ were obtained by normalizing the wall shearing stresses by the free stream dynamic pressure. As discussed in the previous paragraph, the wall shearing stresses obtained using the two different boundary conditions almost collapsed into a single line so that the present computational results are independent of the extent of the far field boundary and the boundary conditions prescribed at the far field boundary. The measured data and the computational results obtained using a curvature correction method (Baskaran, Smits \& Joubert 1987) are also shown in this figure for comparison. It can be seen in Figure 5 that the calculated wall shearing stress for the curved hill is slightly smaller than the measured data near the inlet boundary. This discrepancy is attributed to the inlet boundary condition obtained from a fully developed turbulent boundary layer flow. Apparently, the mass flow rate entering the computational domain prescribed by the velocity profile for a fully developed turbulent flow is smaller than that for the developing velocity profile made turbulent with a trip wire. The influence of the slightly smaller mass flow rate is observed in the wall shearing stress distribution throughout the curved hill. In Figures 5 and 6, "S" represents the separation location and "R" represent the reattachment location. The $M-S$ turbulence model predicted the small reversed flow region near the rear end of the curved hill. On the other hand, the calculated wall shearing stress using the curvature correction method is in close agreement with the measured data. This accurate computational result may due to the use of a boundary layer flow solver which incorporates the measured pressure distribution on the wall (or, equivalently, the external mean flow velocity). However, the curvature 
correction method failed to predict the reversed flow region in the rear end of the curved hill. For the flow over the symmetric airfoil, the present computational result is in excellent agreement with the measured data. This excellent agreement is attributed to the the fact that the mass flow rate of the flat inlet velocity profile is in closer agreement with that of the experiment and to the use of the M-S turbulence model which can describe the turbulence field in a developing boundary layer flow more accurately. The wall shearing stress obtained using the same boundary layer flow solver and the curvature correction method does not compare very well with the measured data. This disagreement may be due to the curvature correction method which can not resolve the strong, developing turbulence field even though the measured pressure (or, the external potential velocity) is used in the boundary layer calculations. Again, the present computational result shows the reversed flow region located near the trailing edge of the airfoil while the curvature correction method fails to predict such a reversed flow region. It is shown in Figure 6 that the separation location for the airfoil compares more favorably with the measured data than it does in the case of flow over the curved hill. The mean velocity profiles for the flow over the curved hill and the airfoil at four downstream locations are compared with the measured data in Figure 7, where the measured mean velocity profiles in physical units were recovered from the measured velocity profiles given in non-dimensional form using the measured wall pressure. In this figure, s'-s-0.074 meters and 0.074 meters represent the correction factor for effective origins of the two flows based on the pressure gradient parameter, see Baskaran, Smits \& Joubert (1987) for details on the effective origin. It can be seen in this figure that the calculated and the measured mean velocity profiles exhibit 
good comparison in general. For the flow over the airfoil, a fluid particle along the symmetry line is brought to zero velocity at the forward stagnation point and a strong adverse pressure gradient is formed in this region. Due to the strong adverse pressure gradient, the velocity in this region is significantly retarded. The computational results show that the mean tangential velocity for the airfoil in this region is smaller than that for the curved hill. The computational results also show that the tangential velocity for the airfoil is slightly larger than that for the curved hill in farther downstream locations. These results simply reflect that the inlet mass flow rate for the airfoil is larger than that for the curved hill in numerical calculations. Note that the measured mean velocity profiles for the airfoil at $s^{\prime}=1.139$ meters and 1.862 meters are smaller than those for the curved hill. The smaller mean velocity for the airfoil in this region is due to the slightly higher pressure distribution in the plateau region of the airfoil which is subjected to a slight experimental uncertainty as discussed previously.

The calculated displacement and momentum thicknesses are compared with the measured data in Figures 8 and 9, respectively. The displacement thickness $\left(\delta^{*}\right)$ and the momentum thickness $(\theta)$ were calculated using equations given as:

$$
\begin{aligned}
\delta^{*} & =\int_{0}^{\infty}\left(1-\frac{U}{U_{p}}\right) d n \\
\theta & =\int_{0}^{\infty} \frac{U}{U_{p}}\left(1-\frac{U}{U_{p}}\right) d n
\end{aligned}
$$


where $U_{p}=U_{p w} e^{k n}$ is the local potential velocity, $U_{p w}-U_{\infty}\left(1-c_{p w}\right)^{1 / 2}$ is the potential velocity on the wall, $k$ is the curvature of the surface, and $n$ is the normal distance from the wall. Eqs. (12) and (13) were used in So \& Mellor (1973) and Baskaran, Smits \& Joubert (1987) to study thin shear layers over curved surfaces, since the classical boundary layer theory and the relationship between the potential velocity $\left(U_{\infty}\right)$ and the wall pressure given as " $\mathrm{P}_{\infty}+0.5 \rho \mathrm{U}_{\infty}{ }^{2}=$ constant" does not hold very well for flows over curved surfaces. It can be seen in these figures that the calculated results and the measured data compare favorably in general. The calculated displacement and momentum thicknesses of the curved hill near the inlet boundary are slightly larger than the measured data. These slight discrepancies are again due to the inlet boundary condition as discussed previously. However, as the flow approaches the curved hill, these discrepancies disappear and the calculated results are in excellent agreement with the measured data. This observation partly shows that the development of the internal layer on the curved hill is only slightly influenced by the approaching external boundary layer flow. It has been shown in Figure 7 that the mean velocity profiles for the curved hill and the airfoil at $s^{\prime}=1.139$ meters are almost identical. However, the displacement thickness and the momentum thickness of the flows over the curved hill and the airfoil, which were obtained from almost the same velocity profiles, are significantly different in the same region near the leading edges as shown in Figures 8 and 9 . These disagreements may due to the use of the boundary layer theory for flows over curved surfaces somewhat beyond its applicable limit. Recall that eqs. (12) and (13) were originally intended to be used for flows over curved surfaces with very small curvature (So \& Mellor 1973). The turbulent flow approaching the 
curved hill is highly retarded due to the strong adverse pressure gradient existing near the leading edge of the curved hill and thus the displacement and the momentum thicknesses are increased significantly in this region. The same flow slightly beyond the leading edge is subjected to far stronger favorable pressure gradient and is accelerated enormously. Thus the displacement and the momentum thicknesses decrease abruptly. In farther downstream, the internal layer is formed gradually and thus these thicknesses grow gradually until the flow is subjected to separation at the rear end of the curved hill. Near the separation point, these integral parameters increase abruptly again. It was suggested in Baskaran, Smits \& Joubert (1987) that the wavy nature of the displacement and momentum thicknesses of the flow over the curved hill might have been caused by the use of the trip wire. However, as discussed in the above, the present computational results suggest that the wavy nature of these integral parameters is inherent to the flow over the curved hill. It is also interesting to note that any turbulence model incorporating wall function methods may not be able to describe the turbulence field over the curved hill adequately partly due to the complex turbulence structure of the internal layer and partly due to the wavy nature of the boundary layer thickness. For example, the optimal distance from the wall where a wall function method can be applied is obscured due to the rapidly varying boundary layer thickness.

The calculated Reynolds stress profiles for the flow over the hill and the flow over the airfoil at a number of downstream locations are shown in Figure 10. The same Reynolds stress profiles in wall coordinates are shown in Figure 11. It can be seen in Figure 10 that the calculated Reynolds stress profile at $s=0.710$ meters is slightly more spread out than the 
measured data. This discrepancy is again attributed to the inlet boundary condition obtained from a fully developed boundary layer flow. At farther downstream locations, the calculated and the measures Reynolds stress profiles are in fair comparison with the measured data qualitatively and quantitatively. The form of the Reynolds stress profile at $s=0.710$ meters is similar to that of a wall-bounded simple shear layer flow and it belongs to the external boundary layer flow. It is shown in Figure 10 that the strength of the Reynolds stress of the external boundary layer flow decays gradually and that of the newly forming internal layer grows rapidly as the fluid travels in the downstream direction. At farther downstream locations, these two Reynolds stress profiles merge together and form a new profile which is similar to that of a wall-bounded simple shear layer flow. Thus the shape of the Reynolds stress profiles in most parts of the curved hill are distinctively different from those found in wall-bounded simple shear layer flows. It can be seen in Figure 11 that there exists a slight difference between the near-wall Reynolds stress profiles of the flow over the curved hill and the flow over the airfoil due to the different upstream turbulence structure. It can also be found that the maximum magnitude of the Reynolds stress is located at $y^{+} \approx 20$. The average physical distance from the wall to this location is approximately equal to $4 \times 10^{-4}$ meters and thus detailed measurement of the turbulence structure in this region can be very difficult. Development of the maximum Reynolds stress along the flow direction is shown in Figure 12. For the flow over the curved hill, a large amount of Reynolds stress is convected downward from the upstream, while the magnitude of the Reynolds stress convected downward in the flow over the airfoil is almost negligible. However, as the flows approach the plateaus of the curved hill and the airfoil, the maximum Reynolds stresses 
of these distinctively different flows become almost the same. Thus it is found that the highly intense turbulence field of the flow over the curved hill is almost independent of the upstream turbulence level and mostly dependent upon the curvature of the curved hill.

The turbulent kinetic energy profiles of the curved hill and the airfoil at a number of downstream locations are shown in Figure 13 and those in wall coordinates are shown in Figure 14. Development of the maximum turbulent kinetic energy along the flow direction is shown in Figure 15. The turbulent kinetic energy profile at $s=0.710$ meters shows that the turbulence intensity of the tripped flow is larger than that of the fully developed boundary layer flow. However, at downstream locations, the calculated turbulent kinetic energy profiles are in good agreement with the measured data. This good agreement at downstream locations indicates that the development of the turbulence field in turbulent flows over strongly curved surfaces depends only siightly on the upstream turbulence intensities. It also indicates that the M-S turbulence model can correctly predict the turbulence fields in a strongly inequilibrium state. It is also shown in Figure 15 that the upstream turbulence intensity of the flow over the curved hill is higher than that of the flow over the curved hill near the inlet boundaries and that the turbulence intensities of these two flows become almost the same at farther downstream locations for the same reason as discussed previously.

\section{CONCLUSIONS AND DISCUSSION}

Detailed computational results for the turbulent flows over a curved hill and a symmetric airfoil obtained using a multiple-time-scale turbulence model were presented. The computational results show that an 
internal layer is developed beneath the external boundary layer. The development of the internal layer depends mostly on the mean flow strain rates distributed over the curved hill and is only slightly influenced by the external boundary layer flow. The curved hill and the airfoil shared the same geometry. The pressure distributions on the walls, the wall shearing stresses, and the mean velocity profiles for the flow over the curved hill and the airfoil were almost the same. Thus the mean flow strain rates are also almost the same in these two flows. The internal layer of the flow over the curved hill, which was caused by almost the same mean flow strain rate as that of the airfoil, was also very similar to the boundary layer flow developed over the symmetric airfoil. It can be seen from these numerical results, as well as from the measured data, that the development of the turbulence field on strongly curved surfaces depends only slightly on the external (or, the upstream) flows and depends mostly on the mean flow strain rate caused by the curved surfaces. Thus the development of the turbulence fields in these two flows are similar to the saturated behavior observed in turbulent flows over strongly curved surfaces .

In the turbulent flow over the curved hill, the pressure gradient changes from adverse to favorable and back to adverse again. The displacement and momentum thicknesses of the boundary layer flow subjected to this pressure gradient increased abruptly near the forward corner of the curved hill, decreased significantly and increased gradually along the curved hill, and then increased abruptly again near the rear end of the curved hill where the flow separates. Thus the wavy nature of these integral parameters as observed in the numerical results and in the measured data is considered to be the inherent nature of the flow over the 
curved hill.

The development of the internal layer was more obvious in the turbulence field (the Reynolds stress profiles and the turbulent kinetic energy profiles) than in the mean velocity profiles. Thus the internal layer can be characterized as a strong turbulence field developing underneath the external boundary layer and in the region very close to the wal1. Development of such a strong turbulence field was caused by the mean flow strain rate. Therefore, development of the internal layer slightly lags that of the mean velocity field.

In the flow over the curved hill, the turbulence intensity of the external boundary layer becomes weaker while that of the internal layer becomes stronger as the fluid flows in the downstream direction. Both the calculated and the measured data showed that the turbulence fields of the external boundary layer and the internal layer merged together in the middle region of the curved hill and formed a new turbulence field which is similar to that of a wall-bounded simple shear layer at the rear end of the curved hill. The internal layer was located very close to the wall and thus development of the wall shearing stress and the flow separation at the rear end of the curved hill were determined solely by the internal layer. The velocity profiles and the wall shearing stress distributions on the curved hill and the airfoil were almost the same. Thus the logarithmic velocity profiles, obtained by non-dimensionalizing the mean velocity profiles using the wall shearing stress, for the curved hill and the airfoil are also very simflar as can be found in Baskaran, Smits \& Joubert (1987). However, the Reynolds stress and the turbulent kinetic energy profiles slightly away from the near-wall region of the curved hill are significantly different from those of the airfoil. From these results, it is found that the 
logarithmic velocity profile alone is not sufficient to characterize the complex turbulent flow over the curved hill. 


\section{REFERENCES}

Baskaran, V., Smits, A. J. \& Joubert, P. N. 1987 A Turbulent Flow over a Curved Hill; Part I Growth of an Internal Boundary Layer, J. Fluid Mech., $182,47$.

Bradbury, L. J. S. 1965 The Structure of a Self-Preserving Turbulent Plane Jet, J. Fluid Mech., 23, 31.

Bradshaw, P. 1969 J. Fluid Mech., 36, 177.

Bradshaw, P. 1973 Effects of Streamline Curvature on Turbulent Flow, AGARDograph 169.

Braza, M., Chassaing, P. \& Ha Minh, H. 1986 Numerical Study and Physical Analysis of the Pressure and Velocity Fields in the Near Wake of a Circular Cylinder, J.Fluid Mech., 165,79.

Chen, H. C. \& Patel, V. C. 1987 Practical Near-Wall Turbulence Models for Complex Flows Including Separation," AIAA paper 87-1300.

Driver, D. M. \& Seegmiller, H. L. 1985 Features of a Reattaching Turbulent Shear Layer in Divergent Channel Flow, J. AIAA, 23, 163.

Gibson, M. M. 1978 An Algebraic Stress and Heat Flux Model for Turbulent Shear Flow with Streamline Curvature, Int. J. Heat Mass Transfer, 21, 1609 .

Gibson, M. M., Jones, W. P. \& Younis, B. A. 1981 Calculation of Turbulent Boundary Layers on Curved Surfaces, Phys. Fluids, 24, 386.

Gillis, J. C. \& Johnston, J. P. 1983 Turbulent Boundary Layer Flow and Structure on a Convex Wall and Its Redevelopment on a Flat Wall, J. Fluid Mech., 135, 123.

Hanjelic, K., Launder, B. E. \& Schiestel, R. 1980 Multiple-Time-Scale Concepts in Turbulent Shear Flows. In Turbulent Shear Flows, Vol. 2, Springer-Verlag, New York. (ed. Bradbury, L. J. S., Durst, F., 
Launder, B. E., Schmidt, F. W. \& Whitelaw, J. H.).

Harlow, F. H. \& Nakayama, P. I. 1968 Transport of Turbulence Energy Decay

Rate, Los Alamos Sci. Lab., LA-3854.

Harris, V. G., Graham, J. A. H. \& Corrsin, S. 1977 Further Experiments in

Nearly Homogeneous Turbulent Shear Flow, J,Fluid Mech., 81, 657.

Irwin, H. P. A. H. 1973 Measurements in a Self-Preserving Plane Wall Jet in

a Positive Pressure Gradient, J. Fluid Mech., 61, 33.

Kim, J., Kline, S. J. \& Johnston, J. P. 1980 Investigation of a Reattaching Turbulent Shear Layer: Flow over a Backward-Facing Step," ASME J. Fluids Engineering, 102, 302 .

Kim, S.-W. \& Chen, C.-P. 1987 A Multiple-Time-Scale Turbulence model Based on Variable Partitioning of the Turbulent Kinetic Energy Spectrum, NASA CR-179222, Also available as AIAA Paper 88-0221, 1988 and to appear in Numerical Heat Transfer.

Kim, S.-W. \& Chen, Y.-S. 1988 A Finite Element Computation of Turbulent Boundary Layer Flows with an Algebraic Stress Turbulence Model, Comput. Meth. App1. Mech. Engrg., 66, 45.

Kim, S.-W. 1988 A Near-Wall Turbulence Model and Its Application to Fully Developed Turbulent Channel and Pipe Flows, NASA TM-101399, Also to appear in Numerical Heat Transfer.

Kim, S.-W. 1989a A Control-Volume Based Reynolds Averaged Navier-Stokes Equation Solver Valid at A11 Flow Velocities, NASA TM-101488.

Kim, S.-W. 1989b Numerical Computation of Shock Wave - Turbulent Boundary Layer Interaction in Transonic Flow over an Axisymmetric Curved Hill, NASA TM-101473.

Klebanoff, P. S. 1955 Characteristics of Turbulence in a Boundary Layer with Zero Pressure Gradient, NACA Report 1247. 
Kline, S. J., Cantwe11, B. J. \& Lilley, G. M. (ed) 1982 Proc 1980-81 AFOSR-HTTM Stanford Conference on Complex Turbulent Flows, Vo1. 1-3, Thermoscience Div., Stanford University.

Laufer, J. 1949 Investigation of Turbulent Flow in a Two-Dimensional Channe1, NACA CR-1053.

Launder, B. E. 1982 A Generalized Algebraic Stress Transport Hypothesis, J. AIAA, $20,436$.

Lumley, J. L. 1983 Turbulence Modelling, ASME J. Applied Mechanics, 50, 1097.

Patankar S. V. 1980 Numerical Heat Transfer and Fluid Flow, McGraw-Hill, New York.

Persen, L. N. 1986 Concepts of Turbulence and CFD Applications, Progress in Aerospace Sciences, 23, 167.

Roback, R. \& Johnson, B. V. 1983 Mass and Momentum Turbulent Transport Experiments with Confined Swirling Coaxial Jets, NASA CR-168252.

Rodi, W. 1972 The Prediction of Free Boundary Layers by Use of a Two-Equation Model of Turbulence, Ph. D. Thesis, University of London, London.

Roshko, A. 1976 Structure of Turbulent Shear Flows: A New Look, I, AIAA, 14,1349 .

So, R. M. C. \& Mellor, G. 1973 Experiments on Convex Curvature Effects in Turbulent Boundary Layers, J. Fluid Mech., 60, 43.

Tennekes, H. \& Lumley, J. L. 1972 A First Course in Turbulence, MIT Press, London.

Tsiolakis, E. P., Krause, E.\& Muller, U. R. 1983 Turbulent Boundary Layer - Wake Interaction. In Turbulent Shear Flows, Vol. 4, Springer-Verlag, New York, (ed. Bradbury, L. J. S., Durst, F., Launder, 
B. E., Schmidt, F. W. \& Whitelaw, J. H.)

Tu, S. W. \& B. R. Ramaprian, B. R. 1983 Fully Developed Periodic Turbulent Pipe Flow, Part I. Main Experimental Results and Comparison with Predictions, J. Fluid Mech., 137, 31.

Vanka, S. P., Chen, B. C. J. \& Sha, W. T. 1980 A Semi-Implicit Calculation Procedure for Flows Described in Boundary-Fitted Coordinate System, Numer. Heat Transfer, 3,1 .

Wolfshtein, M. 1969 The Velocity and Temperature Distribution in

One-Dimensional Flow with Turbulence Augmentation and Pressure Gradient, Int. J. Heat and Mass Transfer, 12, 301.

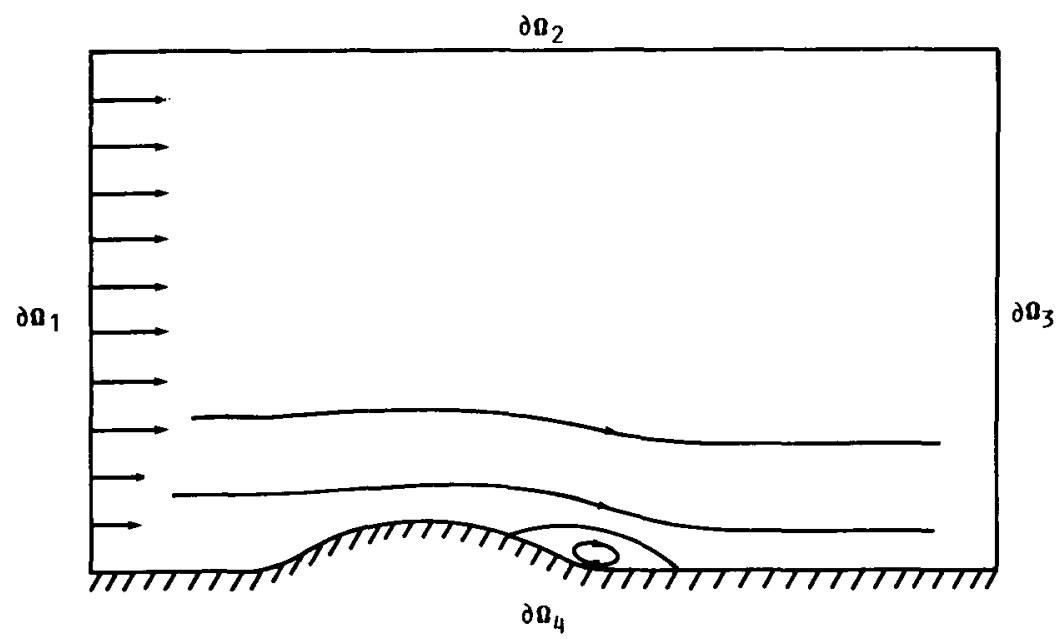

FIGURE 1. - MOMENCLATURE FOR TURBULENT FLOW OVER A CURVED HILL. 


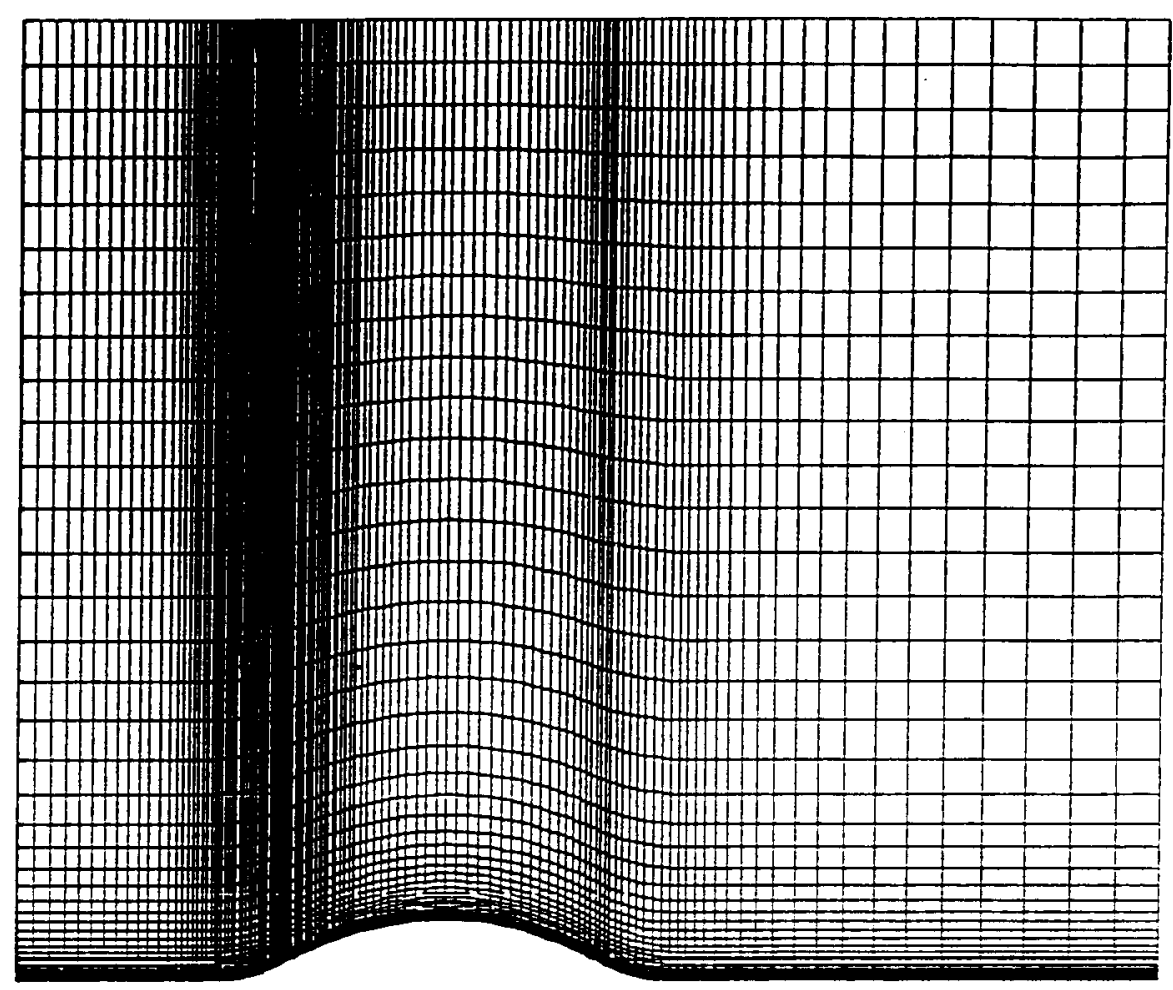

(a) CURVED HILL.

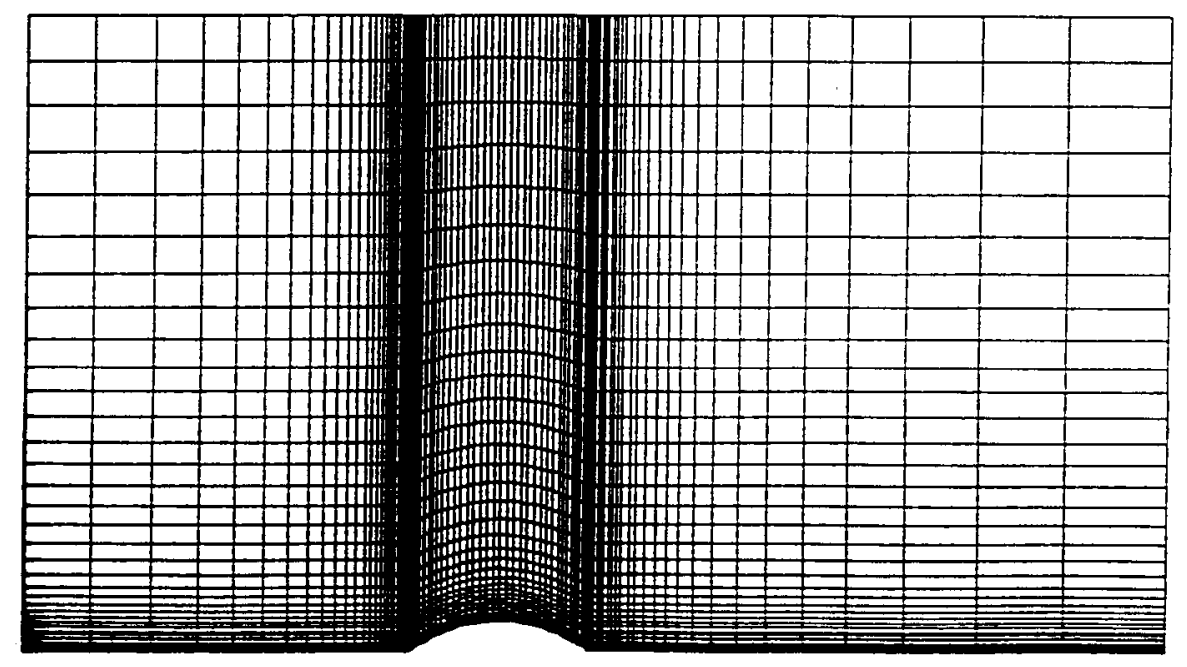

(b) SYMMETRIC AIRFOIL.

FIGURE 2. - DISCRETIZED FLOW DOMAINS. 


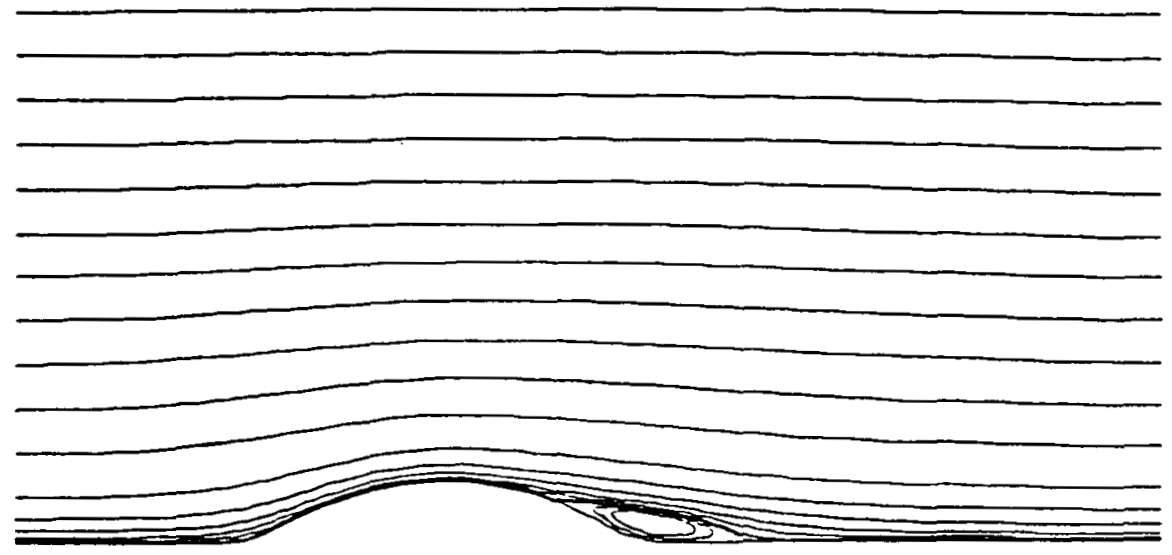

(a) CURVED HILL.

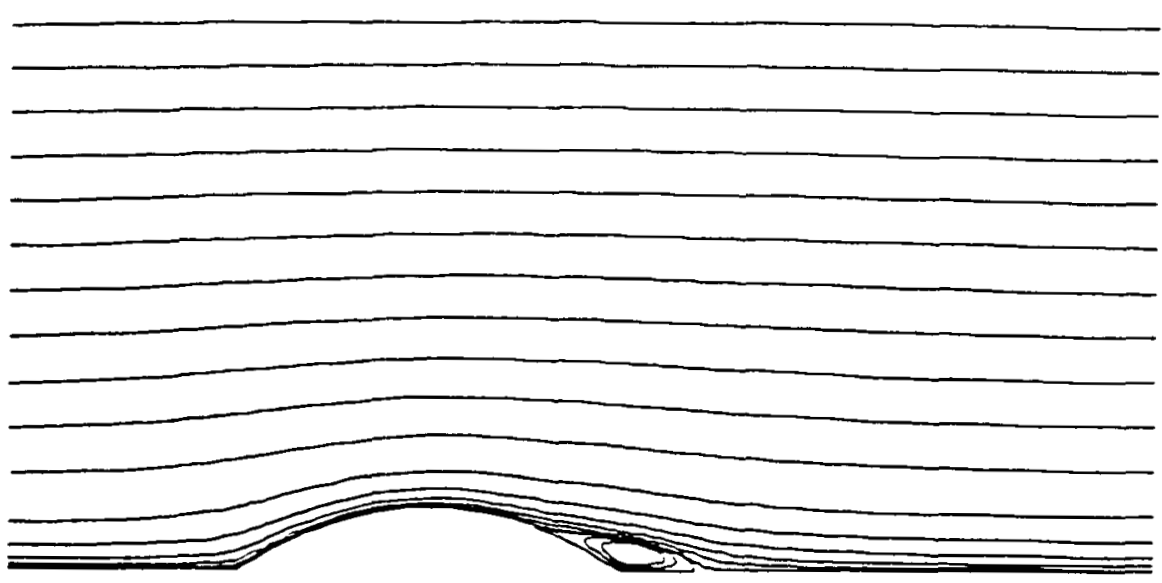

(b) SYMMETRIC AIRFOIL.

FIGURE 3. - STREAML INE CONTOURS. 


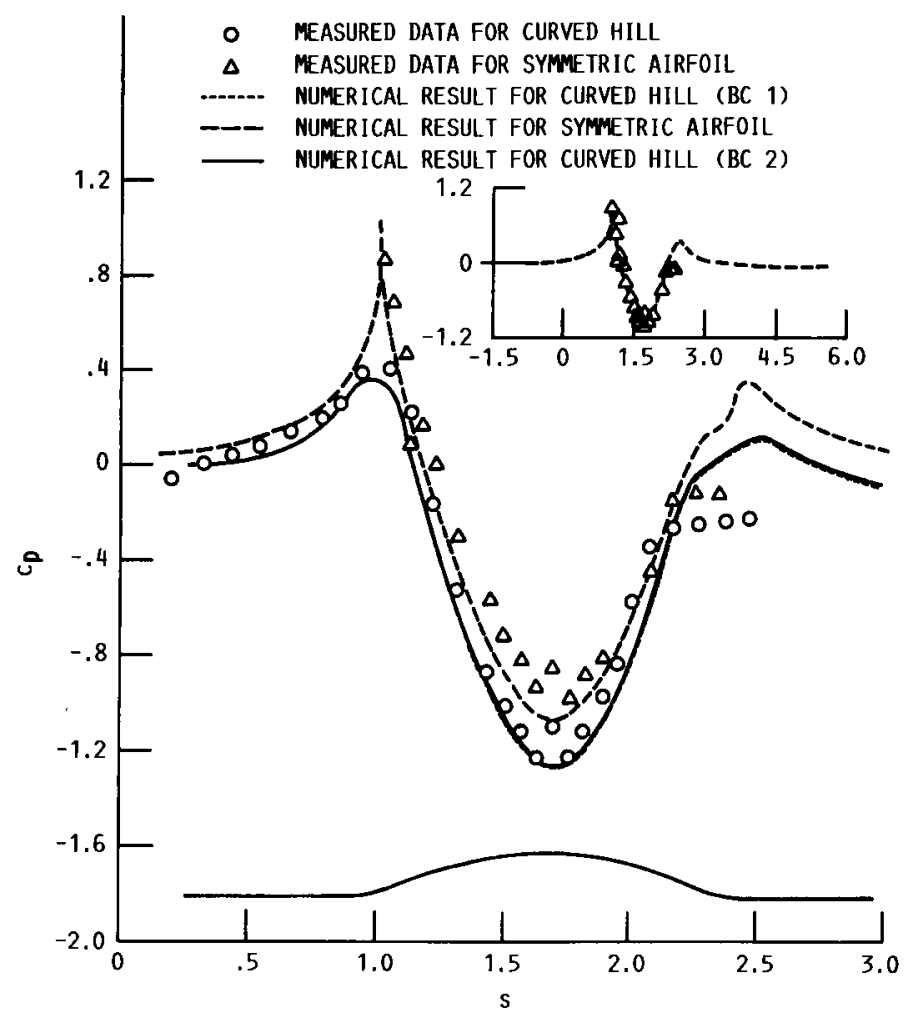

FIGURE 4. - STATIC PRESSURE DISTRIBUTIONS ON THE WALLS.

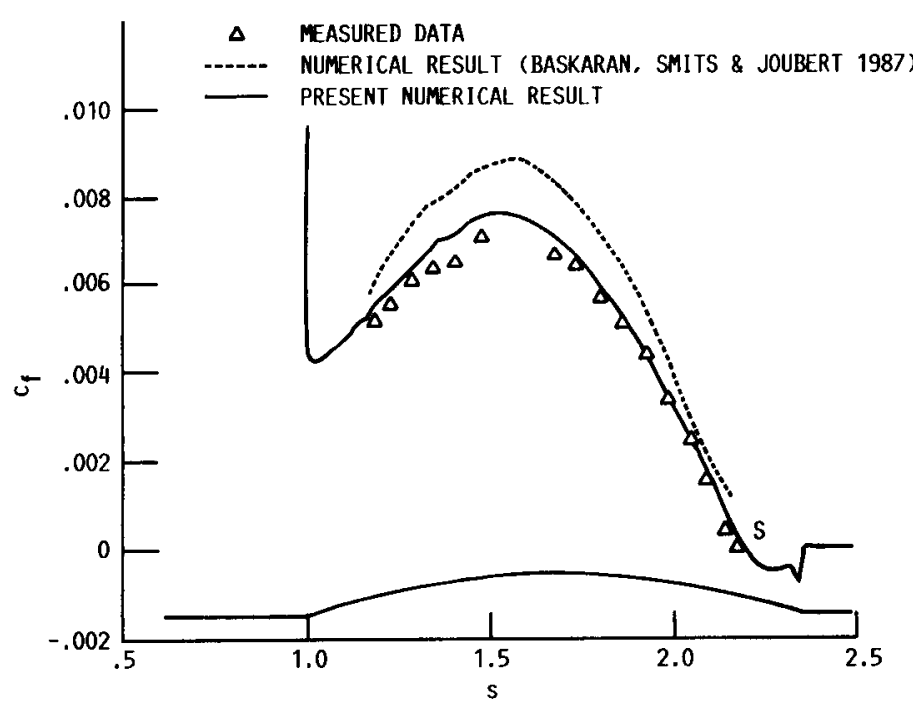

FIGURE 6. - WALL SHEARING STRESS FOR SYMMETRIC AIRFOIL.

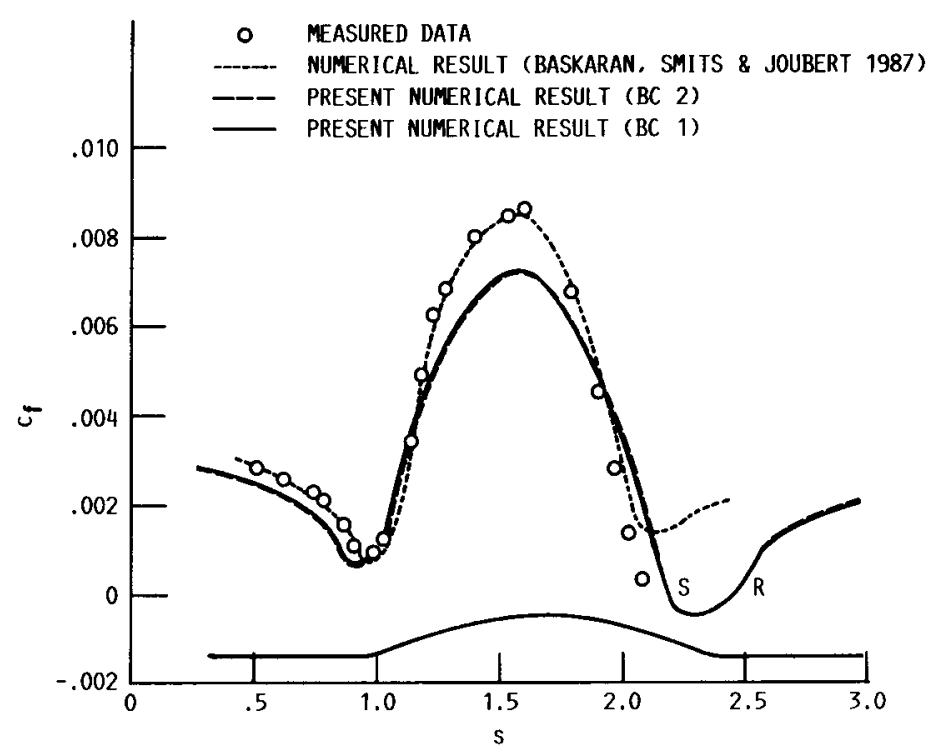

FIGURE 5. - WALL SHEARING STRESS FOR CURVED HILL.

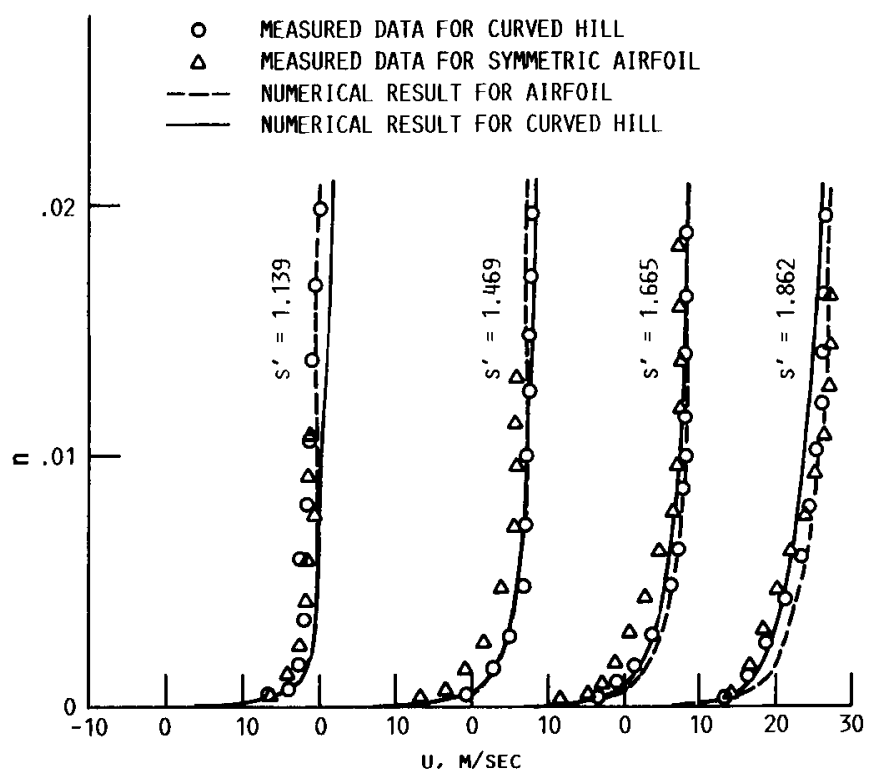

FIGURE 7. - MEAN VELOCITY PROFILES. 


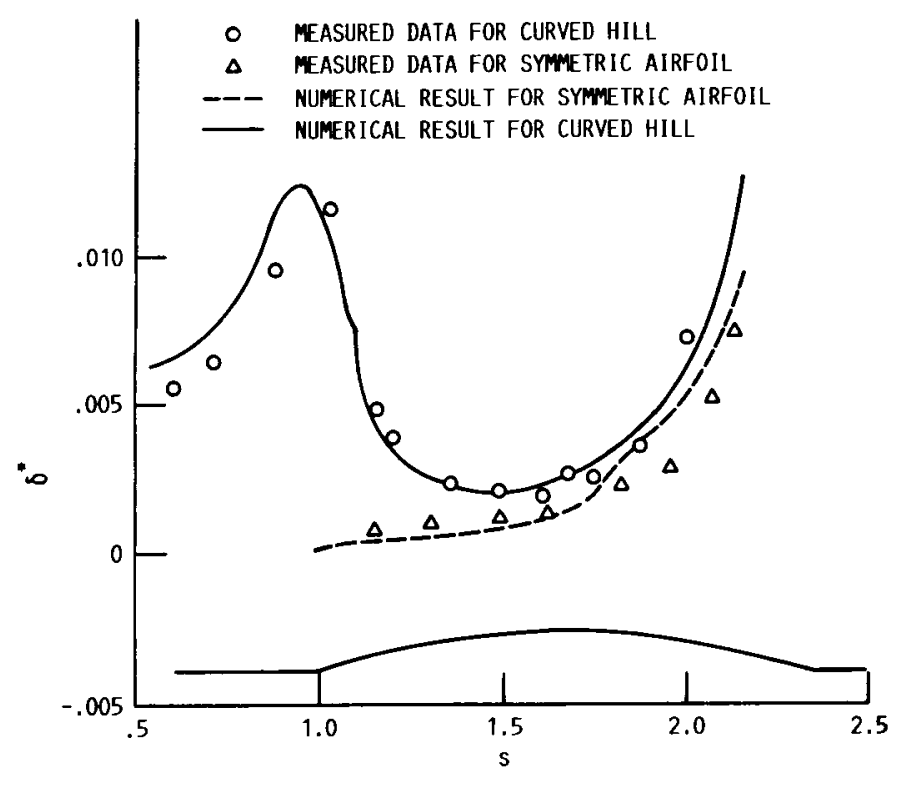

FIGURE 8. - DISPLACEMENT THICKNESS.

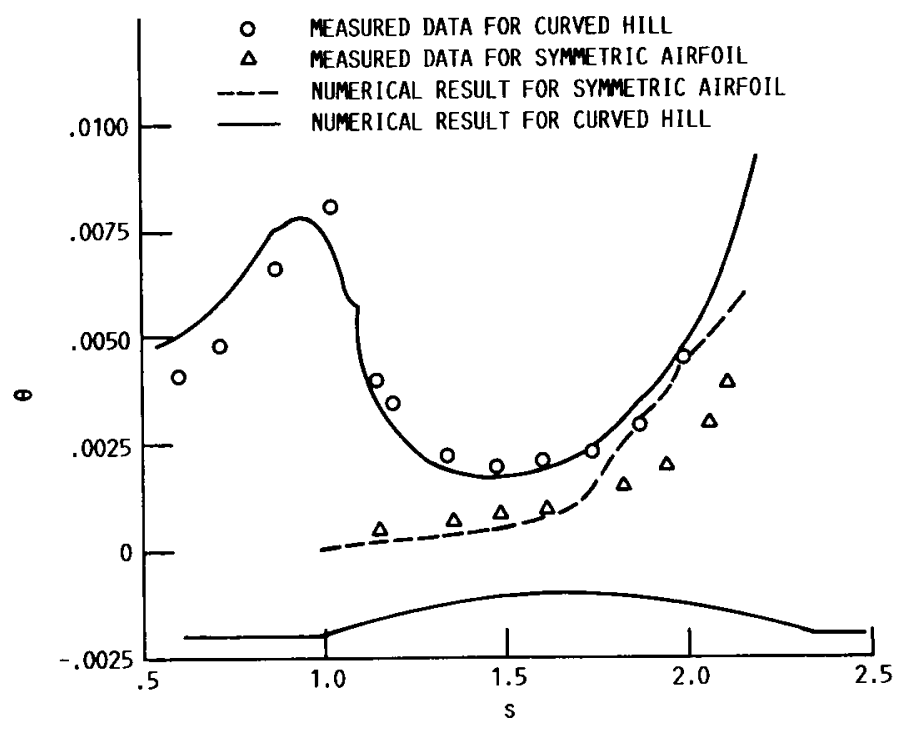

FIGURE 9. - MOHENTUM THICKMESS.

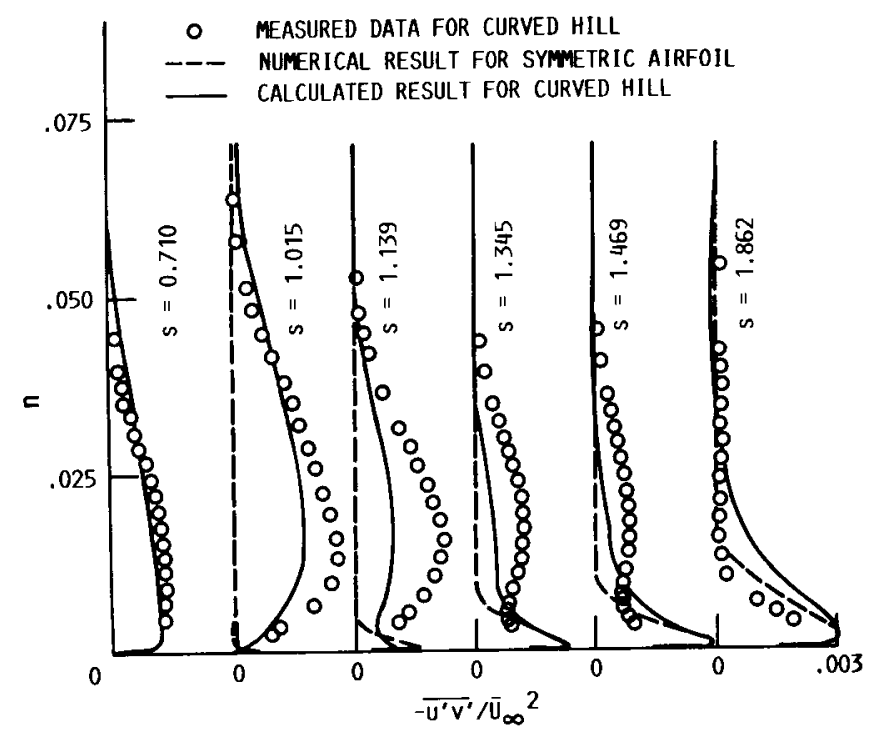

FIGURE 10. - REYMOLDS STRESS PROFILES.

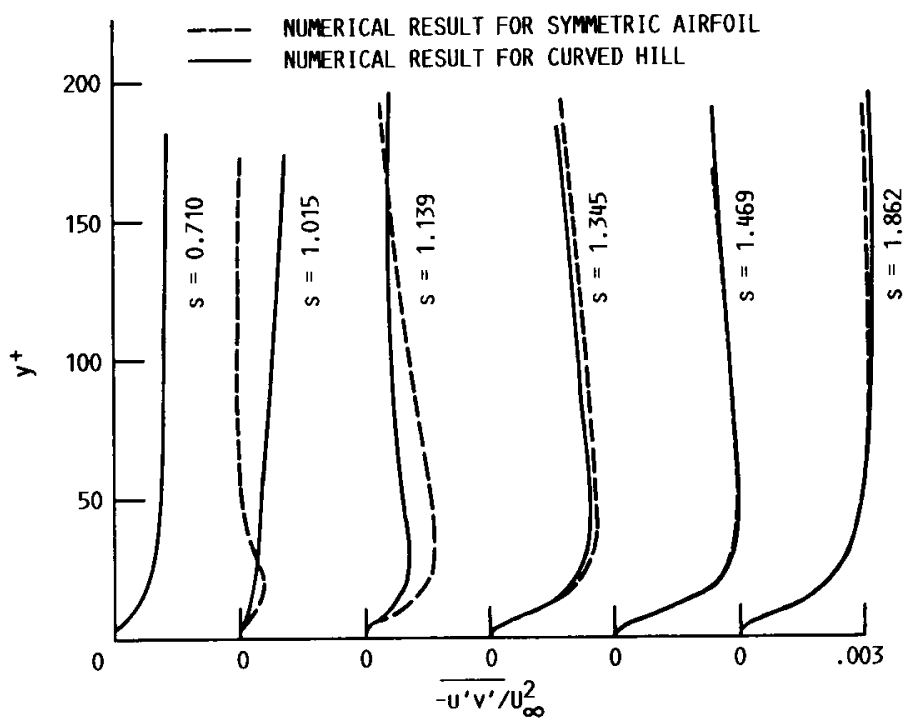

FIGURE 11. - REYMOLDS STRESS IN THE MEAR-MALL REGION. 


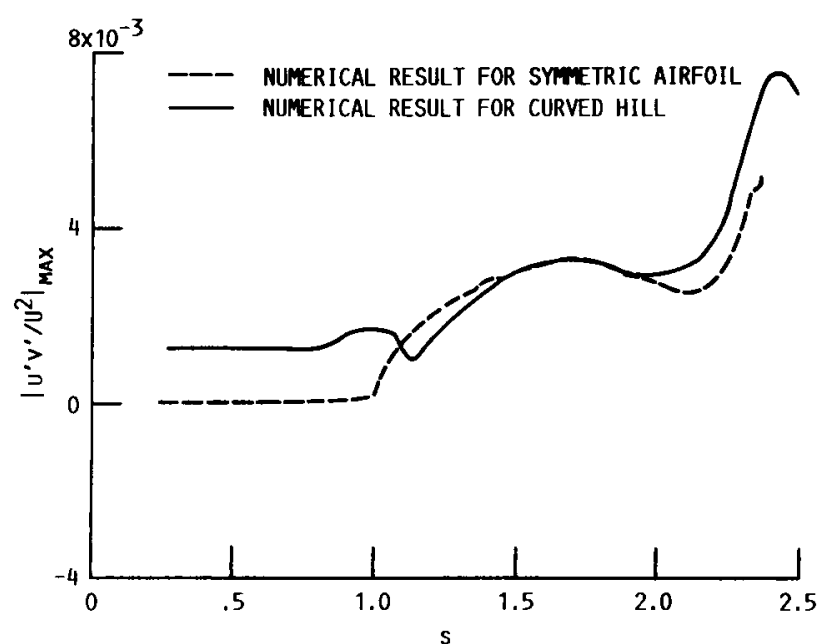

FIGURE 12. - MAXIMUM REYNOLDS STRESS DISTRIBUTIONS ALONG THE FLOW DIRECTION.

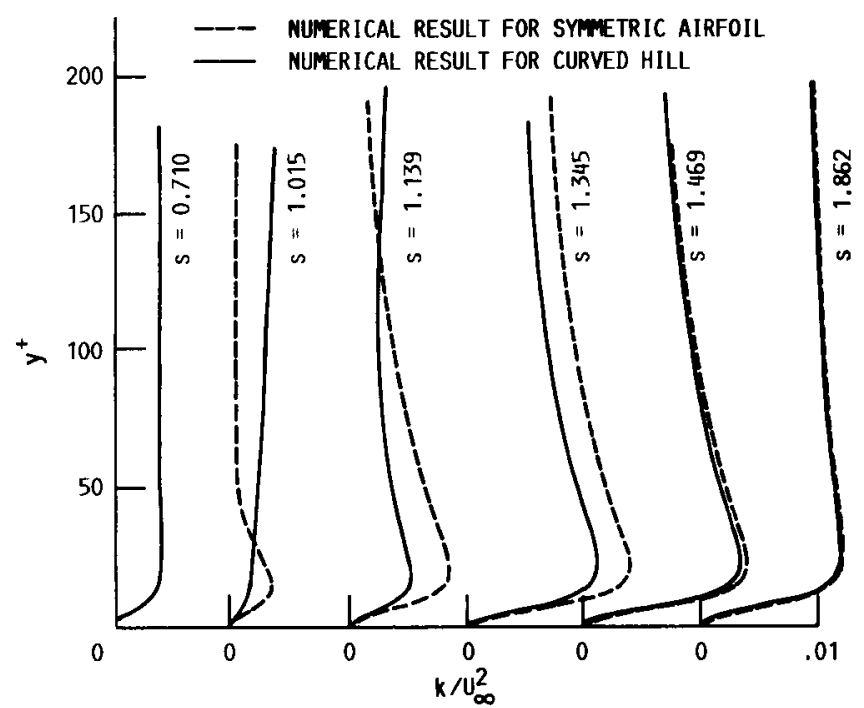

FIGURE 14. - TURBULENT KINETIC ENERGY PROFILES IN THE NEAR-WALL REGION.

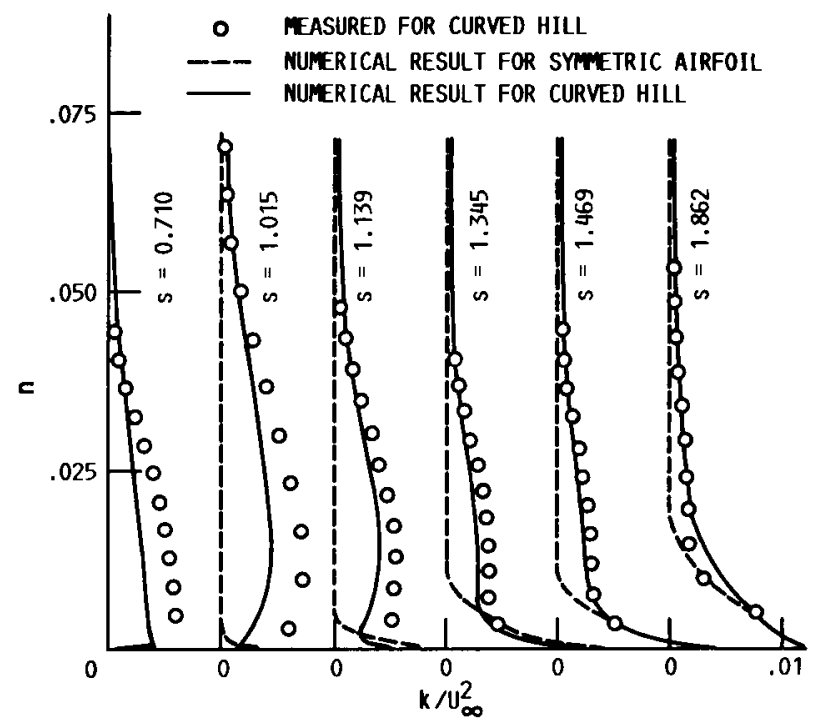

FIGURE 13. - TURBULLENT KINETIC ENERGY PROFILES. NOTATIONS AS IN FIG. 10.

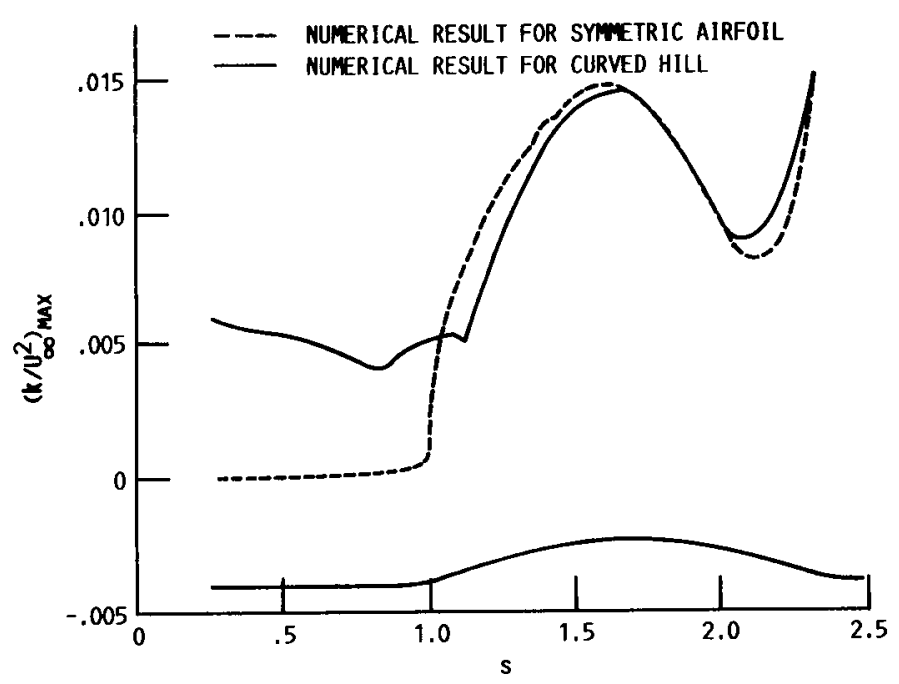

FIGURE 15. - MXIIMM TURBULENT KINETIC ENERGY DISTRIBUTION. 


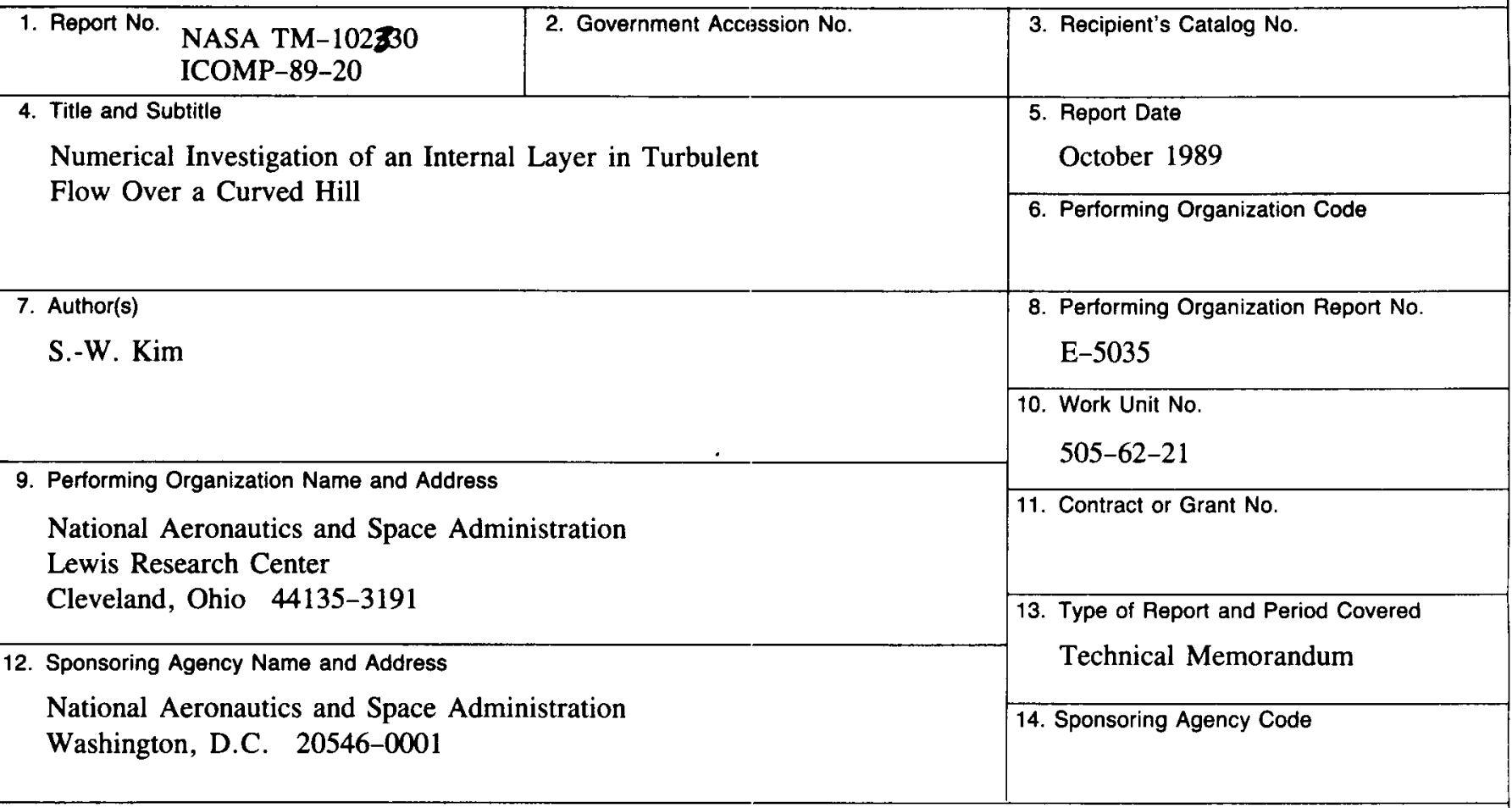

15. Supplementary Notes

S.-W. Kim, Institute for Computational Mechanics in Propulsion, Lewis Research Center, Cleveland, Ohio 44135

(work funded by Space Act Agreement C99066G). Space Act Monitor: Louis A. Povinelli.

16. Abstract

The development of an internal layer in a turbulent boundary layer flow over a curved hill is investigated numerically. The turbulence field of the boundary layer flow over the curved hill is compared with that of a turbulent flow over a symmetric airfoil (which has the same geometry as the curved hill except that the leading and trailing edge plates were removed) to study the influence of the strongly curved surface on the turbulence field. The turbulent flow equations are solved by a control-volume based finite difference method. The turbulence is described by a multiple-time-scale turbulence model supplemented with a near-wall turbulence model. Computational results for the mean flow field (pressure distributions on the walls, wall shearing stresses and mean velocity profiles), the turbulence structure (Reynolds stress and turbulent kinetic energy profiles), and the integral parameters (displacement and momentum thicknesses) compared favorably with the measured data. Computational results show that the internal layer is a strong turbulence field which is developed beneath the external boundary layer and is located very close to the wall. Development of the internal layer was more obviously observed in the Reynolds stress profiles and in the turbulent kinetic energy profiles than in the mean velocity profiles. In this regard, the internal layers is significantly different from wallbounded simple shear layers in which the mean velocity profile characterizes the boundary layer most distinguishably. Development of such an internal layer, characterized by an intense turbulence field, is attributed to the enormous mean flow strain rate caused by the streamline curvature and the strong pressure gradient. In the turbulent flow over the curved hill, the internal layer begin to form near the forward corner of the hill, merges with the external boundary layer, and develops into a new fully turbulent boundary layer as the fluid flows in the downstream direction. For the flow over the symmetric airfoil, the bouridary layer began to form from almost the same location as that of the curved hill, grew in its strength, and formed a fully turbulent boundary layer from mid-part of the airfoil and in the downstream region. Computational results also show that the detailed turbulence structure in the region very close to the wall of the curved hill is almost the same as that of the airfoil in most of the curved regions except near the leading edge. Thus the internal layer of the curved hill and the boundary layer of the airfoil were also almost the same. Development of the wall shearing stress and separation of the boundary layer at the rear end of the curved hill mostly depends on the internal layer and is only slightly influenced by the external boundary layer flow.

\section{Key Words (Suggested by Author(s))}

Streamline curvature, Curvature correction method, Saturated turbulent flow, Multiple-time-scale turbulence model, Control-volume method
18. Distribution Statement

Unclassified - Unlimited

Subject Category 34

19. Security Classif. (of this report)

20. Security Classif. (of this page)

Unclassified

Unclassified

21. No of pages

40
22. Price*

A03 